\title{
Homeostasis model assessment of insulin resistance and the risk of cardiovascular events in middle-aged non-diabetic Japanese men
}

\author{
K. Nakamura • M. Sakurai • K. Miura • Y. Morikawa • \\ M. Ishizaki • K. Yoshita • T. Kido • Y. Naruse • \\ H. Nakagawa
}

Received: 25 January 2010 / Accepted: 27 April 2010/Published online: 26 May 2010

(C) Springer-Verlag 2010

\begin{abstract}
Aims/hypothesis Little is known about the relationship between the HOMA of insulin resistance (HOMA-IR) and the risk of cardiovascular events in Asian populations, which have lower levels of HOMA-IR than Western populations. Accordingly, we determined the predictive value of HOMA-IR for cardiovascular risk in a Japanese
\end{abstract}

K. Nakamura $(\bowtie) \cdot$ M. Sakurai $\cdot$ Y. Morikawa $\cdot$ H. Nakagawa Department of Epidemiology and Public Health,

Kanazawa Medical University,

1-1 Daigaku, Uchinada,

Ishikawa 920-0293, Japan

e-mail:knaka@kanazawa-med.ac.jp

K. Miura

Department of Health Science,

Shiga University of Medical Science,

Otsu, Japan

M. Ishizaki

Department of Social and Environmental Medicine,

Kanazawa Medical University,

Uchinada, Japan

K. Yoshita

Department of Food and Human Health Sciences,

Osaka City University Graduate School of Human Life Science,

Osaka, Japan

T. Kido

School of Health Sciences, College of Medical, Pharmaceutical and Health Sciences, Kanazawa University,

Kanazawa, Japan

\section{Y. Naruse}

Department of Human Science and Fundamental Nursing,

Toyama University School of Nursing,

Toyama, Japan population that was apparently free of diabetes, addressing whether insulin resistance itself increases cardiovascular risk independently of other relevant metabolic disorders. Methods We followed 2,548 non-diabetic men aged 35 to 59 years for 11 years. The hazard ratios for the incidence of cardiovascular events due to increased HOMA-IR were estimated using a Cox proportional hazards model that was adjusted for potential confounding factors.

Results The multivariate-adjusted hazard ratio for cardiovascular events compared with the first quartile of HOMAIR $(\leq 0.66)$ was $1.07(95 \%$ CI $0.44-2.64)$ for the second (HOMA-IR 0.67-1.01), $1.36(0.56-3.28)$ for the third (HOMA-IR 1.02-1.51) and 2.50 (1.02-6.10) for the fourth quartile (HOMA-IR $\geq 1.52$ ). The hazard ratio associated with a one SD (0.61) increment in log-transformed HOMA-IR was 1.51 (1.13-2.02). A similar positive relationship was observed for coronary events and stroke. In addition, the relationship between HOMA-IR and cardiovascular risk was broadly similar in participants with and without hypertension, dyslipidaemia (elevated triacylglycerol and/or reduced HDL-cholesterol), abdominal obesity and current smoking.

Conclusions/interpretation Increased HOMA-IR predicted subsequent cardiovascular events in non-diabetic Japanese men. The association was independent of traditional cardiovascular risk factors and other relevant metabolic disorders.

Keywords Cardiovascular diseases - Coronary heart disease - Epidemiology · Homeostasis model assessment . Insulin resistance $\cdot$ Stroke

\author{
Abbreviation \\ HOMA-IR HOMA of insulin resistance
}




\section{Introduction}

Insulin resistance characterised by decreased sensitivity of tissue to insulin and compensatory elevation in fasting plasma insulin leads not only to abnormal glucose metabolism [1, 2], but also to elevated blood pressure and abnormal lipid profiles such as elevated triacylglycerol and reduced HDL-cholesterol [3-6]. Some investigators have suggested that insulin resistance with compensatory hyperinsulinaemia plays a key role in the clustering of relevant metabolic disorders in the same individual (the metabolic syndrome) [7-10] and that this clustering is a high-risk state for the development of cardiovascular disease [11-14]. However, the contribution of insulin resistance with compensatory hyperinsulinaemia to the development of cardiovascular disease is likely to be independent of abnormal glucose metabolism and other relevant metabolic disorders $[1,6,15-22]$. Since insulin resistance is highly prevalent in the general population $[3,18,23,24]$, it is important to know whether the presence of insulin resistance is an early indicator of increased cardiovascular risk and whether physicians should evaluate insulin resistance to improve overall cardiovascular risk prediction.

The HOMA of insulin resistance (HOMA-IR) is easily available for estimating insulin resistance and is well correlated with estimates of insulin resistance obtained from the euglycaemic-hyperinsulinaemic clamp technique (gold standard) [25, 26]. A number of cohort studies, mainly in Western populations, have examined the relationship between HOMA-IR and the risk of cardiovascular events (including coronary events and stroke) in a general or nondiabetic population [11, 12, 19-21, 27-36]. However, only a few of these studies showed that increased HOMA-IR predicts subsequent cardiovascular events separately from other relevant metabolic disorders [19-21]. In addition, little is known about the relationship between HOMA-IR and the risk of cardiovascular events in Asian populations [35, 36], which have a relatively lower prevalence of obesity and lower levels of HOMA-IR than Western populations [12, 19, $21,24]$. We therefore attempted to determine the predictive value of HOMA-IR for the occurrence of a first-ever cardiovascular event in middle-aged Japanese men who were apparently free of diabetes.

\section{Methods}

Study design and participants The study population consisted of Japanese men who worked for a metal products factory in Toyama prefecture, Japan; this factory employed approximately 4,400 men and 2,600 women. The Industrial Safety and Health Law in Japan requires employers to conduct annual health examinations on all employees.
Examinations include screening tests for traditional cardiovascular risk factors and questionnaires on medical history and lifestyle. Details of this study population have been reported previously [37, 38]. In 1996, 2,952 male employees aged 35 to 59 years, who accounted for approximately $90 \%$ of all male workers of target age, participated in a baseline survey that included a usual health examination and measurement of fasting plasma insulin. The participants were followed-up for 11 years until March 2007. Written informed consent was obtained. The present cohort study was approved by the Institutional Review Committee of Kanazawa Medical University for Ethical Issues.

Of the 2,952 participants, 59 were excluded due to a history of previous cardiovascular events $(n=11)$, missing information at the time of the baseline survey $(n=15)$ or failure to obtain information in the follow-up survey $(n=33)$. To evaluate the true effect of insulin resistance on the occurrence of cardiovascular events independently of abnormal glucose metabolism and to diminish the possibility of inaccurate estimates of insulin resistance from HOMA-IR $[39,40]$, participation in the study was restricted to individuals who were apparently free of diabetes at baseline in order. Thus, 345 additional participants were excluded due to abnormal glucose metabolism defined as fasting glucose $\geq 6.11 \mathrm{mmol} / \mathrm{l}, \mathrm{HbA}_{1 \mathrm{c}} \geq 5.8 \%$ and/or taking medication for diabetes [41]. The remaining 2,548 participants were included in the analyses.

Baseline examination Data collected at study entry included age, medical history, smoking and alcohol drinking habits, leisure-time physical activity and anthropometric indices including waist circumference, blood pressure, serum total cholesterol, HDL-cholesterol, triacylglycerol, fasting plasma glucose, insulin and $\mathrm{HbA}_{1 \mathrm{c}}$. Fasting blood samples were obtained by cubital venipuncture and then shipped to a single laboratory (BML, Toyama, Japan) for analysis. Plasma fasting glucose levels were measured enzymatically using an automatic analyser (GA1140; Kyoto Daiichi Kagaku, Kyoto, Japan). Fasting plasma insulin was measured by radioimmunoassay (Gamma Counter ARC-950; Aloka, Tokyo, Japan). HOMA-IR was calculated using a previously published formula [25]. Other blood chemical markers were also measured using widely accepted methods. Measurements of anthropometric indices and blood pressure were carried out by trained staff. Information on medical history and lifestyle was obtained using a self-administered questionnaire.

Follow-up survey Vital status and the incidence of cardiovascular events were ascertained in March 2007, representing a follow-up period of over 11 years. Questionnaires on medical history in the annual health check-ups and medical certifications for absence due to illness were used to obtain 
information on cardiovascular event history for participants who remained employed at the target factory. Similar questionnaires were sent by mail once a year to retired participants. The medical records of all participants who were thought to have a cardiovascular event were reviewed to confirm the diagnosis.

The diagnostic criteria for myocardial infarction were modified on the basis of those of the Monitoring trends and determinants of cardiovascular disease (MONICA) project conducted by the World Health Organization [42]. Myocardial infarction was defined as typical chest pain with abnormal and persistent Q or QS waves in the electrocardiogram and/or changes in cardiac enzyme activity. Sudden cardiac death was defined as death within $1 \mathrm{~h}$ of onset, a witnessed cardiac arrest or abrupt collapse. Angina pectoris was also included as a coronary event when patients underwent coronary artery angioplasty or bypass surgery. Stroke was defined as a focal neurological disorder with rapid onset, which persisted for at least $24 \mathrm{~h}$ or until death, with supporting evidence from examinations such as computed tomography or magnetic resonance imaging.

The primary outcome in the present study was the incidence of a first-ever cardiovascular event. All such events were classified into two categories: coronary events and stroke. The former included myocardial infarction, sudden cardiac death and angina pectoris requiring an intervention, whereas the latter included cerebral infarction, cerebral haemorrhage, subarachnoid haemorrhage and unspecified stroke.

Statistical analysis Initially, hazard ratios and their corresponding $95 \%$ CIs for the outcomes of interest were calculated for each quartile of HOMA-IR at baseline, with the first quartile serving as the reference. A Cox proportional hazards regression model was used that incorporated the following variables as covariates: age (years), waist circumference $(\mathrm{cm})$, smoking habits (current, former or never smoking), drinking habits (heavy, light, occasional or no drinking), leisure-time physical activity (hard, moderate, light or no activity), systolic blood pressure ( $\mathrm{mmHg}$ ), medication for hypertension (yes or no), log-transformed triacylglycerol $(\mathrm{mmol} / \mathrm{l})$, HDL-cholesterol $(\mathrm{mmol} / \mathrm{l})$, nonHDL-cholesterol (mmol/l), medication for hypercholesterolaemia (yes or no) and $\mathrm{HbA}_{1 \mathrm{c}}(\%)$. Non-HDL-cholesterol was calculated as total cholesterol minus HDL-cholesterol and used as a covariate instead of LDL-cholesterol [43]. Values for triacylglycerol were logarithmically transformed due to their skewed distribution. In addition, the trend between HOMA-IR and the risk of cardiovascular events was explored in a multivariate Cox model with a continuous term for log-transformed HOMA-IR (due to their skewed distribution) instead of HOMA-IR category. We also conducted a similar analysis, in which the reference was the combination of the first and second quartiles of HOMA-IR. Hazard ratios associated with a one SD increment in log-transformed HOMA-IR were also estimated in the Cox model. This approach was applied to fasting insulin, as well as to HOMA-IR, to see whether the association with cardiovascular risk was similar for these two indices.

An analysis was also performed based on previous evidence of the association between HOMA-IR and insulin resistance in a Japanese population. Oimatsu et al. [44] reported that when setting the cut-off value for HOMA-IR at 1.73 in a Japanese population, the sensitivity and specificity for the presence of insulin resistance evaluated by the euglycaemic-hyperinsulinaemic clamp technique were $64.3 \%$ and $78.9 \%$, respectively. Using this evidence as a landmark for grouping HOMA-IR, we divided the participants in our study into the following five groups: (1) HOMA-IR $<1.00$; (2) $1.00 \leq$ HOMA-IR $<1.50$; (3) $1.50 \leq$ HOMA-IR $<2.00$; (4) $2.00 \leq$ HOMA-IR $<2.50$; and (5) $2.50 \leq$ HOMA-IR. Hazard ratios in each HOMA-IR group were calculated in a multivariate Cox model, with the HOMA-IR $<1.00$ group serving as reference.

Finally, analyses were repeated after study participants had been stratified by the presence or absence of: (1) hypertension (defined as systolic blood pressure $\geq 130 \mathrm{mmHg}$, diastolic blood pressure $\geq 85 \mathrm{mmHg}$ and $/$ or taking medication for hypertension); (2) dyslipidaemia (defined as triacylglycerol $\geq 1.69 \mathrm{mmol} / 1$ and/or HDL-cholesterol $<1.03 \mathrm{mmol} / \mathrm{l}$ ); and (3) abdominal obesity (defined as waist circumference $\geq 85 \mathrm{~cm}$ ). The above are based on the Japanese criteria for metabolic syndrome [45] and are all closely linked with insulin resistance with compensatory hyperinsulinaemia [3-6, 24, 46]. This stratification was done to avoid the potential confounding effect of other relevant disorders on cardiovascular risk prediction and to determine whether there was an interaction between each disorder and insulin resistance with regard to risk of cardiovascular events. Similar stratified analyses were also conducted on the basis of smoking status (current smoking or not), because smoking remains a major cardiovascular risk factor in Japanese men [47] and is known to influence plasma insulin levels [48]. The significance of the interaction between increased HOMA-IR and each of the four factors (hypertension, dyslipidaemia, abdominal obesity and smoking) for the risk of cardiovascular events was tested using an interaction term for the categorical variables in the multivariate Cox model.

Statistical analyses were performed using the Statistical Package for the Social Sciences version 12.0J for Windows (SPSS Japan, Tokyo, Japan). All probability values were two-tailed and the significance level was set at $p<0.05$. 


\section{Results}

Characteristics of the study population The baseline characteristics of the 2,548 study participants (mean age 45.0 years) grouped by quartile of HOMA-IR are summarised in Table 1 . The mean age decreased slightly with increasing HOMA-IR. The mean values for body mass index, waist circumference, systolic and diastolic blood pressure, and serum total and non-HDL-cholesterol, as well as the median values for triacylglycerol, fasting plasma glucose and fasting plasma insulin increased with increasing HOMA-IR, whereas the mean value for HDL-cholesterol and the rates of current smoking, light-to-heavy alcohol drinking and moderate-to-hard activity decreased with increasing HOMA-IR.

HOMA-IR and the risk of cardiovascular events The study involved 25,506 person-years of follow-up in 2,548 study participants. The mean overall follow-up period was 10.0 years. During follow-up, 58 first-ever cardiovascular events were recorded, including 25 myocardial infarctions, three sudden cardiac deaths, five cases of angina pectoris with coronary intervention, 13 cerebral infarctions, eight cerebral haemorrhages and four subarachnoid haemorrhages. The crude incidence rate of a first cardiovascular event in the study population was 2.27 per 1,000 person-years.

Compared with the first quartile of HOMA-IR, the second quartile showed little increase in the risk of cardiovascular events, but the third and fourth quartiles showed a gradual trend towards increased risk. The age-adjusted hazard ratio $(95 \% \mathrm{CI})$ was $1.09(0.45-2.62)$ for the second, 1.50 (0.66-3.43) for the third and $2.95(1.41-6.14)$ for the fourth quartile. After further adjustment for traditional cardiovascular risk factors and other metabolic disorders relevant to insulin resistance, the hazard ratio was 1.07 (0.44-2.64), $1.36(0.56-3.28)$ and 2.50 (1.02-6.10), respectively (Fig. 1a). When cardiovascular events were divided into coronary events and stroke, a similar pattern was observed for both event subtypes; the multivariateadjusted hazard ratio comparing the fourth with the first quartile of HOMA-IR was $2.03(0.61-6.75)$ for coronary events and $3.23(0.82-12.79)$ for stroke (Fig. 1b, c). When the first and second quartiles were combined as reference, the multivariate-adjusted hazard ratio comparing the fourth with the first and second quartiles combined was $2.40(1.16-4.94)$ for cardiovascular events (Table 2), 2.27 (0.86-6.00) for coronary events and $2.64(0.89-7.85)$ for stroke.

The trend was significant for all the outcomes, with $p<0.01$ for trend for cardiovascular events, $p=0.04$ for coronary events and $p=0.05$ for stroke. The hazard ratio associated with a one SD (0.61) increment in log-transformed HOMAIR was 1.51 (1.13-2.02) for cardiovascular events (Table 2),
$1.48(1.02-2.14)$ for coronary events and $1.59(1.00-2.54)$ for stroke.

The observed patterns were quite similar between HOMAIR and fasting insulin (pmol/l) for all the outcomes. The multivariate-adjusted hazard ratio for cardiovascular events was $0.91(0.40-2.05)$ for the second $(20.85-34.73 \mathrm{pmo} / \mathrm{l})$, $1.43(0.62-3.34)$ for the third $(34.74-48.62 \mathrm{pmol} / \mathrm{l})$ and 2.60 (1.10-6.15) for the fourth (48.63-506.99 pmol/1) quartile, with the first quartile of fasting insulin (6.95-20.84 pmo/l) serving as the reference. The multivariate-adjusted hazard ratio comparing the fourth with the first quartile of fasting insulin was $1.85(0.57-5.93)$ for coronary events and 4.01 $(1.10-14.67)$ for stroke. The trend was of definite significance or borderline significance for each outcome, with $p<0.01$ for trend for cardiovascular events, $p=0.09$ for coronary events and $p=0.04$ for stroke. The hazard ratio associated with a one SD $(0.58 \mathrm{pmol} / \mathrm{l})$ increment in log-transformed fasting insulin was $1.47(1.10-1.96)$ for cardiovascular events, 1.39 (0.95-2.02) for coronary events and 1.62 (1.03-2.57) for stroke.

In the second approach, the crude incidence rate per 1,000 person-years was 1.56 for HOMA-IR $<1.00(n=1,265), 1.62$ for $1.00 \leq$ HOMA-IR $<1.50(n=620), 2.92$ for $1.50 \leq$ HOMAIR $<2.00(n=349), 3.37$ for $2.00 \leq$ HOMA-IR $<2.50(n=151)$ and 8.15 for $2.50 \leq$ HOMA-IR $(n=163)$, with each group having $20,10,10,5$ and 13 cardiovascular events, respectively. The age-adjusted hazard ratio for cardiovascular events compared with HOMA-IR $<1.00$ was $1.10(0.52$ 2.36) for $1.00 \leq$ HOMA-IR $<1.50,2.07(0.97-4.43)$ for $1.50 \leq$ HOMA-IR $<2.00,2.37(0.89-6.32)$ for $2.00 \leq$ HOMA-IR $<$ 2.50 and $5.83(2.90-11.74)$ for $2.50 \leq$ HOMA-IR; the multivariate-adjusted hazard ratio was $1.07(0.48-2.36)$, 1.95 (0.84-4.53), $2.51(0.85-7.48)$ and 5.54 (2.33-13.15), respectively.

HOMA-IR and the risk of cardiovascular events in patients grouped according to blood pressure, lipids, abdominal obesity or smoking status The associations observed in the overall population were broadly similar in participants with and without hypertension, dyslipidaemia, abdominal obesity or current smoking (Table 2). There was no significant interaction between increased HOMA-IR and any of these four factors with regard to the risk of cardiovascular events ( $p$ values for interaction, see Table 2).

\section{Discussion}

The present cohort study demonstrated a positive relationship between HOMA-IR and the risk of a first-ever cardiovascular event in middle-aged Japanese men who were apparently free of diabetes, adjusting for major cardiovascular risk factors. 
Table 1 Baseline risk characteristics of the 2,548 non-diabetic men participants in Toyama, Japan (1996) grouped by quartile of HOMA-IR

\begin{tabular}{|c|c|c|c|c|c|}
\hline \multirow[t]{2}{*}{ Characteristic } & \multicolumn{4}{|l|}{ HOMA-IR } & \multirow[t]{2}{*}{$p$ value for difference ${ }^{b}$} \\
\hline & $\begin{array}{l}\text { 1st quartile } \\
(0.18-0.66)\end{array}$ & $\begin{array}{l}\text { 2nd quartile } \\
(0.67-1.01)\end{array}$ & $\begin{array}{l}\text { 3rd quartile } \\
(1.02-1.51)\end{array}$ & $\begin{array}{l}\text { 4th quartile } \\
(1.52-18.73)\end{array}$ & \\
\hline Participants $(n)$ & 649 & 629 & 624 & 646 & \\
\hline Age (years) & $45.7 \pm 6.5$ & $45.3 \pm 6.2$ & $44.9 \pm 6.5$ & $44.3 \pm 6.5$ & $<0.01$ \\
\hline HOMA-IR ${ }^{\mathrm{a}}$ & $0.48(0.42-0.62)$ & $0.84(0.76-0.91)$ & $1.24(1.12-1.38)$ & $1.98(1.71-2.52)$ & \\
\hline Height (cm) & $166.9 \pm 6.3$ & $167.7 \pm 6.2$ & $168.2 \pm 5.7$ & $168.4 \pm 5.7$ & $<0.01$ \\
\hline Weight (kg) & $60.3 \pm 7.3$ & $63.9 \pm 7.4$ & $67.1 \pm 7.7$ & $71.0 \pm 8.5$ & $<0.01$ \\
\hline BMI $\left(\mathrm{kg} / \mathrm{m}^{2}\right)$ & $21.6 \pm 2.2$ & $22.7 \pm 2.3$ & $23.7 \pm 2.4$ & $25.0 \pm 2.6$ & $<0.01$ \\
\hline Waist circumference $(\mathrm{cm})$ & $75.3 \pm 6.3$ & $78.5 \pm 6.6$ & $81.4 \pm 6.6$ & $84.7 \pm 7.0$ & $<0.01$ \\
\hline \multicolumn{6}{|l|}{$\begin{array}{l}\text { Cigarette smoking } \\
\text { habits }(\%)\end{array}$} \\
\hline Never & 22.5 & 30.2 & 33.2 & 32.8 & $<0.01$ \\
\hline Former & 7.9 & 12.1 & 11.5 & 14.2 & \\
\hline Current & 69.6 & 57.7 & 55.3 & 52.9 & \\
\hline \multicolumn{6}{|l|}{ Alcohol drinking habits $(\%)$} \\
\hline None & 20.3 & 20.7 & 22.1 & 27.2 & 0.01 \\
\hline Occasional & 28.0 & 32.6 & 31.9 & 31.1 & \\
\hline Light & 28.8 & 28.5 & 28.5 & 25.5 & \\
\hline Heavy & 22.8 & 18.3 & 17.5 & 16.1 & \\
\hline \multicolumn{6}{|l|}{$\begin{array}{l}\text { Leisure-time physical } \\
\text { activity (\%) }\end{array}$} \\
\hline None & 65.5 & 64.1 & 65.7 & 69.7 & 0.02 \\
\hline Light & 16.5 & 21.1 & 21.3 & 19.2 & \\
\hline Moderate & 12.0 & 10.7 & 9.6 & 7.6 & \\
\hline Hard & 6.0 & 4.1 & 3.4 & 3.6 & \\
\hline Systolic BP (mmHg) & $118.8 \pm 13.1$ & $120.6 \pm 13.4$ & $122.5 \pm 14.0$ & $124.9 \pm 14.3$ & $<0.01$ \\
\hline Diastolic BP (mmHg) & $74.6 \pm 9.9$ & $76.2 \pm 10.2$ & $77.0 \pm 10.6$ & $78.8 \pm 10.4$ & $<0.01$ \\
\hline $\begin{array}{l}\text { Medication for } \\
\text { hypertension (\%) }\end{array}$ & 2.9 & 4.3 & 4.5 & 7.9 & $<0.01$ \\
\hline $\begin{array}{l}\text { Serum total } \\
\text { cholesterol }(\mathrm{mmol} / \mathrm{l})\end{array}$ & $5.07 \pm 0.79$ & $5.32 \pm 0.84$ & $5.33 \pm 0.89$ & $5.43 \pm 0.85$ & $<0.01$ \\
\hline $\begin{array}{l}\text { Serum non-HDL- } \\
\text { cholesterol }(\mathrm{mmol} / \mathrm{l})\end{array}$ & $3.51 \pm 0.82$ & $3.81 \pm 0.85$ & $3.96 \pm 0.91$ & $4.16 \pm 0.86$ & $<0.01$ \\
\hline $\begin{array}{l}\text { Hypercholesterolaemia } \\
\text { medication (\%) }\end{array}$ & 0.5 & 0.8 & 2.1 & 1.4 & 0.04 \\
\hline $\begin{array}{l}\text { Serum triacylglycerol } \\
(\mathrm{mmol} / \mathrm{l})^{\mathrm{a}}\end{array}$ & $0.90(0.68-1.24)$ & $1.02(0.77-1.42)$ & $1.22(0.89-1.70)$ & $1.53(1.07-2.15)$ & $<0.01$ \\
\hline $\begin{array}{l}\text { Serum HDL- } \\
\text { cholesterol }(\mathrm{mmol} / \mathrm{l})\end{array}$ & $1.56 \pm 0.42$ & $1.51 \pm 0.40$ & $1.38 \pm 0.35$ & $1.27 \pm 0.33$ & $<0.01$ \\
\hline $\begin{array}{l}\text { Fasting plasma } \\
\text { glucose }(\mathrm{mmol} / \mathrm{l})^{\mathrm{a}}\end{array}$ & $4.77(4.50-4.94)$ & $5.00(4.61-5.27)$ & $5.00(4.77-5.27)$ & $5.16(4.88-5.55)$ & $<0.01$ \\
\hline $\begin{array}{l}\text { Fasting plasma } \\
\text { insulin }(\mathrm{pmol} / \mathrm{l})^{\mathrm{a}}\end{array}$ & $13.89(13.89-20.84)$ & $27.78(20.84-27.78)$ & $41.67(34.73-41.67)$ & $62.51(48.62-76.40)$ & $<0.01$ \\
\hline $\mathrm{HbA}_{1 \mathrm{c}}(\%)$ & $4.99 \pm 0.33$ & $5.01 \pm 0.32$ & $5.00 \pm 0.34$ & $5.03 \pm 0.33$ & 0.12 \\
\hline
\end{tabular}

Values are expressed as mean $\pm \mathrm{SD}$, median (interquartile range) or per cent of participants in the respective category

${ }^{\mathrm{a}}$ Median is presented due to a skewed distribution

${ }^{\mathrm{b}}$ One-way analysis of variance, Kruskal-Wallis test or $\chi^{2}$ test to compare each risk characteristic among the quartiles of HOMA-IR

Similar positive relationships were observed for coronary events and stroke. This pattern was broadly similar regardless of the presence or absence of other relevant metabolic disorders (hypertension and dyslipidaemia), abdominal obe- sity and smoking, with no evidence of an interaction effect between increased HOMA-IR and each of these four factors on the risk of cardiovascular events. To the best of our knowledge, this is the first prospective survey that shows a 

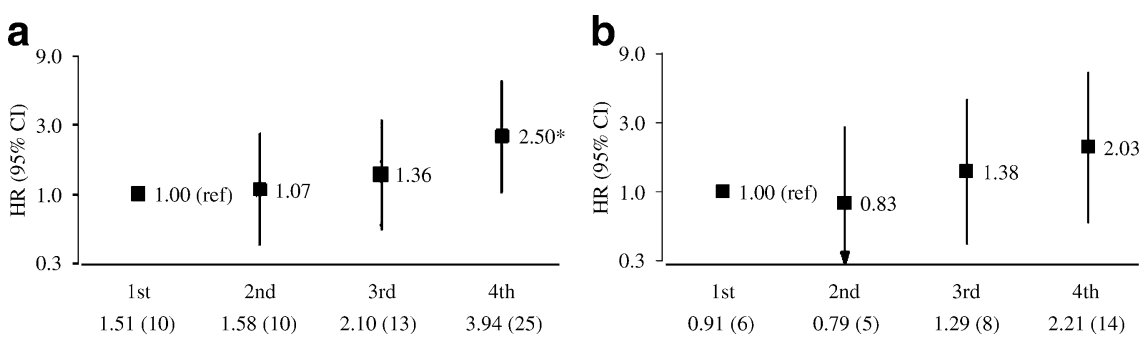

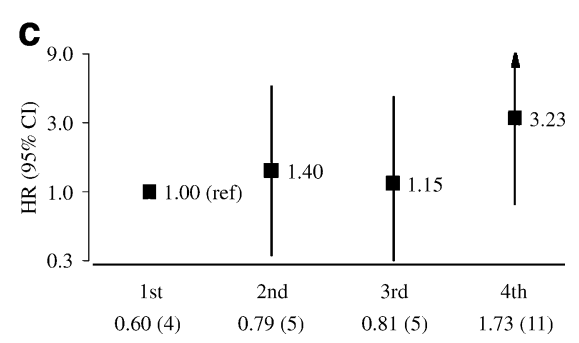

Fig. 1 Hazard ratios for the incidence of (a) cardiovascular events, (b) coronary events and (c) stroke in each quartile of HOMA-IR in 2,548 men over 11 years of follow-up (1996-2007). A Cox proportional hazards regression model was used with adjustment for age, waist circumference, smoking habits, drinking habits, leisure-time physical activity, systolic blood pressure, medication for hypertension, serum
non-HDL-cholesterol, medication for hypercholesterolaemia, log-serum triacylglycerol, serum HDL-cholesterol and $\mathrm{HbA}_{1 \mathrm{c}}$. The ranges of the first $(n=649)$, second $(n=629)$, third $(n=624)$ and fourth $(n=646)$ quartiles of HOMA-IR were $0.18-0.66,0.67-1.01,1.02-1.51$ and 1.52 18.73 , respectively. Values, $x$-axes are crude incidence rates per 1,000 person-years ( $n$ events). $y$-Axes are $\log _{3}$ scale. ${ }^{*} p<0.05$. ref, reference significantly positive relationship between HOMA-IR and the risk of coronary events and stroke in an Asian population, avoiding the potential confounding effect of other relevant metabolic disorders on the risk of cardiovascular events. Although a previous Chinese study examined the relationship between HOMA-IR and the risk of cardiovascular events, that study reported a positive trend, which did not reach statistical significance [36].

Hedblad et al. [19] reported that non-diabetic individuals with the 75th percentile value of the distribution of HOMAIR ( $\geq 2.12$ for men, $\geq 1.80$ for women) of their study population had a significantly higher risk of myocardial infarction than those without these HOMA-IR values, after adjustment for traditional risk factors including fasting glucose. In addition, the Bruneck study [20] reported a similar relationship between HOMA-IR and the risk of cardiovascular events. Furthermore, the San Antonio Heart Study [21] reported that non-diabetic individuals with HOMA-IR $\geq 2$ (which was close to the median value) were at increased risk of coronary artery disease and stroke compared with those with HOMA-IR $<2$. Our results are consistent with the findings of these previous Western studies. An important finding of our study was that increased HOMA-IR can predict subsequent coronary events and stroke in Asians, in whom stroke is the predominant subtype of cardiovascular event and the ratio of ischaemic stroke:haemorrhagic stroke differs from that in Whites [49]. In addition, our data suggest an apparent increase in the risk of cardiovascular events with an HOMA-IR of about 1.5, although the cardiovascular risk remains unchanged below 1.5. Interestingly, our findings support a previous Japanese study, which suggested that a HOMA-IR value of 1.73 was the appropriate cut-off level for insulin resistance [44]. However, further studies are required to provide more detailed information on this issue.

Our stratified analyses further emphasise that insulin resistance with compensatory hyperinsulinaemia has an effect on development of the diseases studied that is distinct from that of other relevant metabolic disorders. In theory, even isolated insulin resistance without any other relevant metabolic disorders may predict subsequent coronary events and stroke. Consequently, measures to reverse insulin resistance in addition to the management of traditional cardiovascular risk factors may improve the overall cardiovascular risk profile, particularly in nondiabetic individuals. In addition, insulin resistance and abdominal obesity may play independent roles, at least in part, in the development of cardiovascular disease, although obesity is closely associated with insulin resistance [24, 46]. Our observations are consistent with the findings of the San Antonio Heart Study [21]. However, the present study did not elucidate the underlying mechanism for the possible causal relationship between insulin resistance with compensatory hyperinsulinaemia and cardiovascular events. It is also unlikely that smoking and insulin resistance have a synergistic effect on the development of cardiovascular disease.

Our study has several limitations. First, as our study participants consisted solely of male workers in one factory, caution should be exercised when generalising our results. Second, only participants who were apparently free of diabetes at baseline were included in the analyses. This inclusion was based on fasting glucose $<6.11 \mathrm{mmol} / \mathrm{l}$ and $\mathrm{HbA}_{1 \mathrm{c}}<5.8 \%$ [41], because we had no data on plasma glucose and insulin after glucose loading. Third, no information was available on other factors that affect fasting insulin, e.g. the presence of an insulin-producing tumour. Finally, coronary events included only cases of angina pectoris requiring coronary intervention; medication-managed cases of angina pectoris were excluded. Furthermore, we were not able to divide stroke into ischaemic and haemorrhagic types in our study due to the relatively small numbers of each event.

In conclusion, our data suggest that HOMA-IR is a useful index for prediction of subsequent coronary events and stroke in a non-diabetic Japanese male population. In addition, insulin resistance with compensatory hyperinsulinaemia is 
Table 2 Hazard ratios for the incidence of cardiovascular events in the third and fourth quartiles of HOMA-IR compared with the combination of the first and second quartiles in 2,548 non-diabetic men over 11 years of follow-up (1996-2007)

\begin{tabular}{|c|c|c|c|c|c|}
\hline \multirow[t]{2}{*}{ Variable } & \multicolumn{3}{|c|}{ HOMA-IR by quartile } & \multirow{2}{*}{$\begin{array}{l}\text { Log-HOMA-IR } \\
1 \mathrm{SD}(0.61) \\
\text { increment }\end{array}$} & \multirow{2}{*}{$\begin{array}{l}p \text { value } \\
\text { for interaction }\end{array}$} \\
\hline & $\begin{array}{l}1 \mathrm{st}+2 \mathrm{nd} \\
(0.18-1.01)\end{array}$ & $\begin{array}{l}3 \mathrm{rd} \\
(1.02-1.51)\end{array}$ & $\begin{array}{l}\text { 4th } \\
(1.52-18.73)\end{array}$ & & \\
\hline \multicolumn{6}{|l|}{ Overall } \\
\hline Events/participants $(n / n)$ & $20 / 1,278$ & $13 / 624$ & $25 / 646$ & & \\
\hline Crude rate per 1,000 person-years & 1.54 & 2.10 & 3.94 & & \\
\hline Multivariate-adjusted HR $(95 \% \mathrm{CI})^{\mathrm{a}}$ & 1.00 (reference) & $1.31(0.63-2.73)$ & $2.40(1.16-4.94)$ & $1.51(1.13-2.02)$ & \\
\hline \multicolumn{6}{|l|}{ Absence of hypertension } \\
\hline Events/participants $(n / n)$ & $9 / 886$ & $3 / 393$ & $14 / 362$ & & \\
\hline Crude rate per 1,000 person-years & 1.00 & 0.77 & 3.91 & & \\
\hline Multivariate-adjusted HR $(95 \% \mathrm{CI})^{\mathrm{a}}$ & 1.00 (reference) & $0.68(0.18-2.61)$ & $3.09(1.11-8.62)$ & $1.34(0.90-1.99)$ & \\
\hline Presence of hypertension ${ }^{\mathrm{b}}$ & & & & & 0.42 \\
\hline Events/participants $(n / n)$ & $11 / 392$ & $10 / 231$ & $11 / 284$ & & \\
\hline Crude rate per 1,000 person-years & 2.80 & 4.37 & 3.98 & & \\
\hline Multivariate-adjusted HR $(95 \% \mathrm{CI})^{\mathrm{a}}$ & 1.00 (reference) & $1.84(0.73-4.64)$ & $1.80(0.64-5.08)$ & $1.69(1.08-2.64)$ & \\
\hline \multicolumn{6}{|l|}{ Absence of dyslipidaemia } \\
\hline Events/participants $(n / n)$ & $15 / 1,061$ & $6 / 426$ & $14 / 322$ & & \\
\hline Crude rate per 1,000 person-years & 1.39 & 1.40 & 4.43 & & \\
\hline Multivariate-adjusted HR $(95 \% \mathrm{CI})^{\mathrm{a}}$ & 1.00 (reference) & $0.81(0.29-2.23)$ & $3.02(1.22-7.44)$ & $1.69(1.16-2.47)$ & \\
\hline Presence of dyslipidaemia ${ }^{\mathrm{b}}$ & & & & & 0.26 \\
\hline Events/participants $(n / n)$ & $5 / 217$ & $7 / 198$ & $11 / 324$ & & \\
\hline Crude rate per 1,000 person-years & 2.33 & 3.66 & 3.45 & & \\
\hline Multivariate-adjusted HR $(95 \% \mathrm{CI})^{\mathrm{a}}$ & 1.00 (reference) & $1.89(0.58-6.20)$ & $1.65(0.51-5.35)$ & $1.19(0.76-1.87)$ & \\
\hline \multicolumn{6}{|l|}{ Absence of abdominal obesity } \\
\hline Events/participants $(n / n)$ & $17 / 1,114$ & $9 / 433$ & $11 / 331$ & & \\
\hline Crude rate per 1,000 person-years & 1.50 & 2.08 & 3.38 & & \\
\hline Multivariate-adjusted HR $(95 \% \mathrm{CI})^{\mathrm{a}}$ & 1.00 (reference) & $1.26(0.54-2.97)$ & $1.96(0.82-4.66)$ & $1.47(1.01-2.13)$ & \\
\hline Presence of abdominal obesity ${ }^{\mathrm{b}}$ & & & & & 0.91 \\
\hline Events/participants $(n / n)$ & $3 / 164$ & $4 / 191$ & $14 / 315$ & & \\
\hline Crude rate per 1,000 person-years & 1.88 & 2.14 & 4.54 & & \\
\hline Multivariate-adjusted HR $(95 \% \mathrm{CI})^{\mathrm{a}}$ & 1.00 (reference) & $1.21(0.26-5.58)$ & $3.85(0.93-15.93)$ & $1.72(1.04-2.84)$ & \\
\hline \multicolumn{6}{|l|}{ Absence of current smoking } \\
\hline Events/participants $(n / n)$ & $4 / 463$ & $1 / 279$ & $10 / 304$ & & \\
\hline Crude rate per 1,000 person-years & 0.85 & 0.36 & 3.22 & & \\
\hline Multivariate-adjusted HR $(95 \% \mathrm{CI})^{\mathrm{a}}$ & 1.00 (reference) & $0.48(0.05-4.58)$ & $4.60(1.11-19.17)$ & $2.06(1.20-3.54)$ & \\
\hline Presence of current smoking & & & & & 0.76 \\
\hline Events/participants $(n / n)$ & $16 / 815$ & $12 / 345$ & $15 / 342$ & & \\
\hline Crude rate per 1,000 person-years & 1.94 & 3.52 & 4.51 & & \\
\hline Multivariate-adjusted HR $(95 \% \mathrm{CI})^{\mathrm{a}}$ & 1.00 (reference) & $1.56(0.70-3.46)$ & $1.79(0.76-4.22)$ & $1.38(0.97-1.94)$ & \\
\hline
\end{tabular}

Data are presented for the total study population (overall) and also grouped according to characteristics as indicated

${ }^{a}$ Cox proportional hazards regression model with multivariate adjustment for age, waist circumference, smoking habits, drinking habits, leisure-time physical activity, systolic blood pressure, medication for hypertension, serum non-HDL-cholesterol, medication for hypercholesterolaemia, log-serum triacylglycerol, serum HDL-cholesterol and $\mathrm{HbA}_{1 \mathrm{c}}$

${ }^{\mathrm{b}}$ Definitions based on the Japanese criteria for metabolic syndrome; hypertension was defined as systolic blood pressure $\geq 130 \mathrm{mmHg}$, diastolic blood pressure $\geq 85 \mathrm{mmHg}$ and/or taking medication for hypertension; dyslipidaemia was defined as triacylglycerol $\geq 1.69 \mathrm{mmol} / 1$ and/or HDL-cholesterol $<1.03 \mathrm{mmol} / 1$; abdominal obesity was defined as waist circumference $\geq 85 \mathrm{~cm} \mathrm{[45]}$

${ }^{\mathrm{c}}$ The significance of the interaction effect between increased HOMA-IR and each of the four factors on the risk of cardiovascular events was tested using an interaction term for the categorical variables in the Cox model 
likely to have an effect on the development of cardiovascular disease separately from other relevant metabolic disorders. Given that HOMA-IR is calculated after the assessment of traditional cardiovascular risk factors and the measurement of fasting insulin, HOMA-IR could provide additional information that could improve overall prediction of cardiovascular risk.

Acknowledgements The present study was supported by the Japan Arteriosclerosis Prevention Fund and by research grants from the Ministry of Health, Labour and Welfare (Comprehensive Research on Cardiovascular and Life-Style Related Disease: H17-Kenko-007, H18Junkankitou [Seishuu]-Ippan-012, and H20-Junkankitou [Seishuu]Ippan-013, and -021 and 22110601).

Duality of interest The authors declare that there is no duality of interest associated with this manuscript.

\section{References}

1. Facchini FS, Hua N, Abbasi F, Reaven GM (2001) Insulin resistance as a predictor of age-related diseases. J Clin Endocrinol Metab 86:3574-3578

2. Bonora E, Kiechl S, Willeit J et al (2004) Population-based incidence rates and risk factors for type 2 diabetes in white individuals: the Bruneck study. Diabetes 53:1782-1789

3. Zavaroni I, Bonora E, Pagliara M et al (1989) Risk factors for coronary artery disease in healthy persons with hyperinsulinemia and normal glucose tolerance. $\mathrm{N}$ Engl $\mathrm{J}$ Med 320:702-706

4. Mitchell BD, Haffner SM, Hazuda HP, Valdez R, Stern MP (1992) The relation between serum insulin levels and 8-year changes in lipid, lipoprotein, and blood pressure levels. Am J Epidemiol 136:12-22

5. Haffner SM, Valdez RA, Hazuda HP, Mitchell BD, Morales PA, Stern MP (1992) Prospective analysis of the insulin-resistance syndrome (syndrome X). Diabetes 41:715-722

6. Yip J, Facchini FS, Reaven GM (1998) Resistance to insulinmediated glucose disposal as a predictor of cardiovascular disease. J Clin Endocrinol Metab 83:2773-2776

7. Reaven GM (1988) Banting lecture 1988. Role of insulin resistance in human disease. Diabetes 37:1595-1607

8. DeFronzo RA, Ferrannini E (1991) Insulin resistance. A multifaceted syndrome responsible for NIDDM, obesity, hypertension, dyslipidemia, and atherosclerotic cardiovascular disease. Diab Care 14:173-194

9. Alberti KG, Zimmet PZ (1998) Definition, diagnosis and classification of diabetes mellitus and its complications. Part 1: diagnosis and classification of diabetes mellitus. Provisional report of a WHO consultation. Diabet Med 15:539553

10. Balkau B, Charles MA (1999) Comment on the provisional report from the WHO consultation. European Group for the Study of Insulin Resistance (EGIR). Diabet Med 16:442-443

11. Isomaa B, Almgren P, Tuomi T et al (2001) Cardiovascular morbidity and mortality associated with the metabolic syndrome. Diab Care 24:683-689

12. Meigs JB, Rutter MK, Sullivan LM, Fox CS, D'Agostino RB Sr, Wilson PW (2007) Impact of insulin resistance on risk of type 2 diabetes and cardiovascular disease in people with metabolic syndrome. Diab Care 30:1219-1225
13. Lakka HM, Laaksonen DE, Lakka TA et al (2002) The metabolic syndrome and total and cardiovascular disease mortality in middle-aged men. JAMA 288:2709-2716

14. Ninomiya T, Kubo M, Doi Y et al (2007) Impact of metabolic syndrome on the development of cardiovascular disease in a general Japanese population: the Hisayama study. Stroke 38:2063-2069

15. Zethelius B, Lithell H, Hales CN, Berne C (2005) Insulin sensitivity, proinsulin and insulin as predictors of coronary heart disease. A population-based 10-year, follow-up study in 70-year old men using the euglycaemic insulin clamp. Diabetologia 48:862-867

16. Pyörälä M, Miettinen H, Laakso M, Pyörälä K (2000) Plasma insulin and all-cause, cardiovascular, and noncardiovascular mortality: the 22-year follow-up results of the Helsinki Policemen Study. Diab Care 23:1097-1102

17. Kiyohara Y, Fujishima M (1997) Insulin resistance and cardiovascular disease incidence in a elderly Japanese population: the Hisayama study. Jpn J Geriatr 34:360-364 (article in Japanese)

18. Fujiwara T, Saitoh S, Takagi S et al (2005) Development and progression of atherosclerotic disease in relation to insulin resistance and hyperinsulinemia. Hypertens Res 28:665-670

19. Hedblad B, Nilsson P, Engström G, Berglund G, Janzon L (2002) Insulin resistance in non-diabetic subjects is associated with increased incidence of myocardial infarction and death. Diabet Med 19:470-475

20. Bonora E, Kiechl S, Willeit J et al (2007) Insulin resistance as estimated by homeostasis model assessment predicts incident symptomatic cardiovascular disease in Caucasian subjects from the general population: the Bruneck study. Diab Care 30:318324

21. Hanley AJG, Williams K, Stern MP, Haffner SM (2002) Homeostasis model assessment of insulin resistance in relation to the incidence of cardiovascular disease: the San Antonio Heart Study. Diab Care 25:1177-1184

22. Barr EL, Cameron AJ, Balkau B et al (2010) HOMA insulin sensitivity index and the risk of all-cause mortality and cardiovascular disease events in the general population: the Australian Diabetes, Obesity and Lifestyle Study (AusDiab) study. Diabetologia 53:79-88

23. Bonora E, Kiechl S, Willeit J et al (1998) Prevalence of insulin resistance in metabolic disorders: the Bruneck Study. Diabetes 47:1643-1649

24. Ohnishi H, Saitoh S, Ura N et al (2002) Relationship between insulin resistance and accumulation of coronary risk factors. Diabetes Obes Metab 4:388-393

25. Matthews DR, Hosker JP, Rudenski AS, Naylor BA, Treacher DF, Turner RC (1985) Homeostasis model assessment: insulin resistance and beta-cell function from fasting plasma glucose and insulin concentrations in man. Diabetologia 28:412-419

26. Bonora E, Targher G, Alberiche M et al (2000) Homeostasis model assessment closely mirrors the glucose clamp technique in the assessment of insulin sensitivity: studies in subjects with various degrees of glucose tolerance and insulin sensitivity. Diab Care 23:57-63

27. Resnick HE, Jones K, Ruotolo G et al (2003) Insulin resistance, the metabolic syndrome, and risk of incident cardiovascular disease in nondiabetic American Indians: the Strong Heart Study. Diab Care 26:861-867

28. Robins SJ, Rubins HB, Faas FH et al (2003) Insulin resistance and cardiovascular events with low HDL cholesterol: the Veterans Affairs HDL Intervention Trial (VA-HIT). Diab Care 26:1513-1517

29. Alssema M, Dekker JM, Nijpels G, Stehouwer CD, Bouter LM, Heine RJ (2005) Proinsulin concentration is an independent predictor of all-cause and cardiovascular mortality: an 11-year follow-up of the Hoorn Study. Diab Care 28:860-865 
30. Rutter MK, Meigs JB, Sullivan LM, D'Agostino RB Sr, Wilson PW (2005) Insulin resistance, the metabolic syndrome, and incident cardiovascular events in the Framingham Offspring Study. Diabetes 54:3252-3257

31. Dekker JM, Girman C, Rhodes T et al (2005) Metabolic syndrome and 10-year cardiovascular disease risk in the Hoorn Study. Circulation 112:666-673

32. Onat A, Hergenç G, Türkmen S, Yazici M, Sari I, Can G (2006) Discordance between insulin resistance and metabolic syndrome: features and associated cardiovascular risk in adults with normal glucose regulation. Metabolism 55:445-452

33. Jeppesen J, Hansen TW, Rasmussen S, Ibsen H, Torp-Pedersen C, Madsbad S (2007) Insulin resistance, the metabolic syndrome, and risk of incident cardiovascular disease: a population-based study. J Am Coll Cardiol 49:2112-2119

34. Jeppesen J, Hansen TW, Olsen MH et al (2008) C-reactive protein, insulin resistance and risk of cardiovascular disease: a populationbased study. Eur J Cardiovasc Prev Rehabil 15:594-598

35. Adachi H, Hirai Y, Tsuruta M, Fujiura Y, Imaizuml T (2001) Is insulin resistance or diabetes mellitus associated with stroke? An 18-year follow-up study. Diabetes Res Clin Pract 51:215-223

36. Chien KL, Hsu HC, Su TC, Chen MF, Lee YT, Hu FB (2008) Fasting and postchallenge hyperglycemia and risk of cardiovascular disease in Chinese: the Chin-Shan Community Cardiovascular Cohort study. Am Heart J 156:996-1002

37. Sakurai M, Miura K, Takamura T et al (2006) Gender differences in the association between anthropometric indices of obesity and blood pressure in Japanese. Hypertens Res 29:75-80

38. Sakurai M, Miura K, Nakamura K et al (2009) Relationship between abdominal obesity, accumulation of metabolic abnormalities and risk of cardiovascular disease: an 11-year follow-up of middle-aged Japanese men. Jpn J Cardiovasc Dis Prev 44:1-9 (article in Japanese)

39. Kang ES, Yun YS, Park SW et al (2005) Limitation of the validity of the homeostasis model assessment as an index of insulin resistance in Korea. Metabolism 54:206-211
40. Sakai K, Nakamaru K, Miyagawa K, Yamaguchi K (2007) Simple indices of insulin resistance and their limits in clinical application. J Japan Diab Soc 50:725-730 (article in Japanese)

41. The Japan Diabetes Society (2008) Guideline for diabetes treatment 2008-2009. Bunkodo, Tokyo (book in Japanese)

42. Tunstall-Pedoe H, Kuulasmaa K, Amouyel P, Arveiler D, Rajakangas AM, Pajak A (1994) Myocardial infarction and coronary deaths in the World Health Organization MONICA Project. Registration procedures, event rates, and case-fatality rates in 38 populations from 21 countries in four continents. Circulation 90:583-612

43. Friedewald WT, Levy RI, Fredrickson DS (1972) Estimation of the concentration of low-density lipoprotein cholesterol in plasma, without use of the preparative ultracentrifuge. Clin Chem 18:499502

44. Oimatsu H, Saitoh S, Ura N, Shimamoto K (2000) A practical index for evaluation of insulin resistance. J Japan Diab Soc 43:205-213 (article in Japanese)

45. No authors listed (2005) Definition and the diagnostic standard for metabolic syndrome - Committee to Evaluate Diagnostic Standards for Metabolic Syndrome. J Jpn Soc Int Med 94:794-809 (article in Japanese)

46. Ludvik B, Nolan JJ, Baloga J, Sacks D, Olefsky J (1995) Effect of obesity on insulin resistance in normal subjects and patients with NIDDM. Diabetes 44:1121-1125

47. Hozawa A, Okamura T, Murakami Y et al (2007) Joint impact of smoking and hypertension on cardiovascular disease and all-cause mortality in Japan: NIPPON DATA80, a 19-year follow-up. Hypertens Res 30:1169-1175

48. Rönnemaa T, Rönnemaa EM, Puukka P, Pyörälä K, Laakso M (1996) Smoking is independently associated with high plasma insulin levels in nondiabetic men. Diab Care 19:1229-1232

49. Ueshima H, Sekikawa A, Miura K et al (2008) Cardiovascular disease and risk factors in Asia: a selected review. Circulation 118:2702-2709 